\title{
Mirizzi Syndrome: Diagnosis and Management of a Challenging Biliary Disease
}

\author{
Gennaro Clemente (DD , ${ }^{1}$ Andrea Tringali, ${ }^{2}$ Agostino M. De Rose $(\mathbb{D}),{ }^{1}$ Elena Panettieri, ${ }^{1}$ \\ Marino Murazio, ${ }^{1}$ Gennaro Nuzzo, ${ }^{1}$ and Felice Giuliante ${ }^{1}$ \\ ${ }^{1}$ Department of Surgical Sciences, Hepatobiliary Unit, Fondazione Policlinico Universitario A. Gemelli IRCCS, \\ Catholic University of Sacred Hearth, Rome, Italy \\ ${ }^{2}$ Department of Surgical Sciences, Surgical Endoscopy Unit, Fondazione Policlinico Universitario A. Gemelli IRCCS, \\ Catholic University of Sacred Hearth, Rome, Italy
}

Correspondence should be addressed to Gennaro Clemente; gennaro.clemente@unicatt.it

Received 26 April 2018; Revised 11 July 2018; Accepted 1 August 2018; Published 12 August 2018

Academic Editor: Olivier Barbier

Copyright ( $) 2018$ Gennaro Clemente et al. This is an open access article distributed under the Creative Commons Attribution License, which permits unrestricted use, distribution, and reproduction in any medium, provided the original work is properly cited.

\begin{abstract}
Background. Mirizzi syndrome is a condition difficult to diagnose and treat, representing a particular "challenge" for the biliary surgeon. The disease can mimic cancer of the gallbladder, causing considerable diagnostic difficulties. Furthermore, it increases the risk of intraoperative biliary injury during cholecystectomy. The aim of this study is to point out some particular aspects of diagnosis and treatment of this condition. Methods. The clinical records of patients with Mirizzi syndrome, treated in the last five years, were reviewed. Clinical data, cholangiograms, preoperative diagnosis, operative procedures, and early and late results were examined. Results. Eighteen consecutive patients were treated in the last five years. Presenting symptoms were jaundice, pain, and cholangitis. Preoperative diagnosis of Mirizzi syndrome was achieved in 11 patients, while 6 had a diagnosis of gallbladder cancer and 1 of Klatskin tumor. Seventeen patients underwent surgery, including cholecystectomy in 8 cases, bile duct repair over Ttube in 3 cases, and hepaticojejunostomy in 4 cases. Two cases (11.1\%) of gallbladder cancer associated with the Mirizzi syndrome were incidentally found: a patient underwent right hepatectomy and another patient was unresectable. The overall morbidity rate was $16.6 \%$. There was no postoperative mortality. An ERCP with stent insertion was required in three cases after surgery. Sixteen patients were asymptomatic at a mean distance of 24 months (range: 6-48) after surgery. Conclusions. Mirizzi syndrome requires being treated by an experienced biliary surgeon after a careful assessment of the local situation and anatomy. The preoperative placement of a stent via ERCP can simplify the surgical procedure.
\end{abstract}

\section{Introduction}

Mirizzi syndrome was firstly reported by Pablo Luis Mirizzi (1893-1964), one of the major biliary surgeons in the past century. Mirizzi was born in Cordoba (Argentina) from Italian parents. He spent all his academic career in his hometown, where he left a deep mark $[1,2]$. He is mainly known for conceiving and performing the first intraoperative cholangiography in 1931, a procedure that had a strong impact on biliary surgery of the XX century. Mirizzi first described his syndrome in 1948 [3], presenting the case of a patient with a big stone impacted in the gallbladder infundibulum, causing jaundice by extrinsic compression of the common bile duct (CBD) with a productive inflammation extending from the gallbladder to the CBD. This condition is an important complication of gallbladder stones and requires a differential diagnosis from gallbladder cancer involving the CBD. In some cases, the stone erodes from the gallbladder into the CBD determining a fistula; consequently, the stone is located in a single cavity formed by Hartmann's pouch and the CBD (Figure 1). In 1982 McSherry et al. named this condition "type II Mirizzi syndrome" [4], almost 20 years after Mirizzi's death. Type II represents the possible evolution of the properly mentioned Mirizzi syndrome (type I). In 1989 Csendes et al. [5] classified type II Mirizzi syndrome in three subgroups (II, III, and IV), considering the entity of the involvement of the 


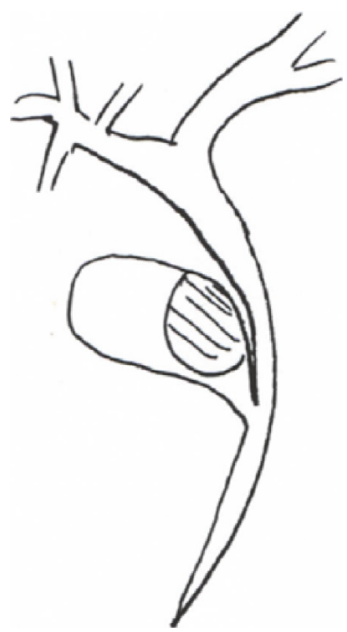

(a)

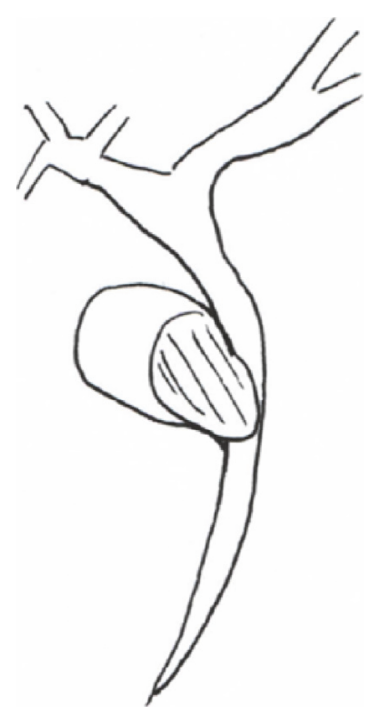

(b)

FIGURE 1: (a) Type I Mirizzi syndrome: a big stone impacted in the gallbladder infundibulum cause jaundice by extrinsic compression of the CBD; (b) type II Mirizzi syndrome: the stone is located in a single cavity formed by gallbladder and CBD.

CBD. In type II of Csendes classification, fistula involves $1 / 3$ of the CBD circumference, in type III it involves the $2 / 3$, and in the type IV the CBD is no more recognizable and represents a whole entity with the gallbladder. Recently, Béltran et al. [6], reporting several cases of Mirizzi syndrome associated with cholecysto-enteric fistula, classified these cases as type $\mathrm{V}$, divided into two subgroups: (a) without gallstone ileus and (b) with gallstone ileus. Later, in response to a letter by SolisCaxaj [7], Béltran [8, 9] simplified the classification of the syndrome identifying only three types:

(i) Type I, "classic" Mirizzi syndrome.

(ii) Type II cholecystocholedochal fistula with two subtypes: (a) diameter less than $50 \%$ of the CBD and (b) diameter more than $50 \%$ of the CBD.

(iii) Type III, with cholecysto-enteric fistula without (a) or with (b) gallstone ileus.

However, many authors continue to use the first classification of Csendes [5] which, by allowing a better distinction about the entity of the CBD wall involved in the fistula, could be useful to establish the right preoperative workup and surgical management.

This need for an accurate classification is related to the high frequency of the syndrome in South America, where it occurs in about $5 \%$ of gallstones, while in Western countries its incidence is about $1 \%$ [10].

Mirizzi syndrome is a condition difficult to diagnose and treat, representing a particular challenge for the biliary surgeon aware of the risk for the patient. The syndrome can mimic gallbladder cancer, but it also represents a precancerous condition, consequently causing considerable diagnostic difficulties. At the same time, because of the significant increase in the risk of intraoperative biliary injury during cholecystectomy, it is a very dangerous condition. At present, a standard treatment of Mirizzi syndrome is not yet well defined, due to the heterogeneous clinical presentation. Surgical treatment should be planned after a careful assessment of the local situation and anatomy. During the past five years we observed some consecutive cases of Mirizzi syndrome, which led us to review this field in an attempt to point out some particular aspects of diagnosis and treatment of this challenging condition.

\section{Materials and Methods}

Detailed clinical records of patients with diagnosis at discharge of Mirizzi syndrome, treated in our unit between January 1st, 2012, and December 31st, 2016, were reviewed. The following data were considered: sex and age of the patients; presenting symptoms and previous treatments; preoperative radiological investigations; preoperative diagnosis; surgical procedures performed; postoperative course (morbidity and mortality); findings at pathological examination. Followup data, obtained by direct clinical observation, laboratory findings (liver function tests), and need for further treatment, were also recorded.

\section{Results}

In the last 5 years, 18 consecutive cases of Mirizzi syndrome were treated in our unit and they represented $1.54 \%$ of 1.165 cholecystectomies performed in the same period. Eleven patients were male and 7 were female with a mean age of 63.4 years (range: 25-90 years). Presenting symptoms were obstructive jaundice in 14 patients, colic pain in 3 patients, and acute cholangitis in one patient. Preoperative imaging included ultrasonography (US) in all cases, Computerized Tomography (CT) in 12 patients, and Magnetic Resonance Imaging (MRI) in 10 patients. Fourteen patients underwent a preoperative ERCP; in all cases a stricture was found and, consequently, one or more stents were positioned in the CBD. 
TABLE 1: Clinical data summarized.

\begin{tabular}{|c|c|c|c|c|c|c|c|}
\hline $\mathrm{Pt}$ & Sex & Age & Symptom & Preop. Imaging & Preop. diagnosis & Treatment & Final diagnosis \\
\hline 1 & $\mathrm{M}$ & 64 & Cholangitis & US, ERCP & Mirizzi & Hepatico-jejunostomy & Mirizzi type II \\
\hline 2 & $\mathrm{M}$ & 79 & Jaundice & US,CT,ERCP & Gallbladder cancer & Cholecystectomy & M. type I \\
\hline 3 & $\mathrm{M}$ & 82 & Pain & US, MRI,ERCP & Mirizzi & Cholecystectomy & M. type I \\
\hline 4 & $\mathrm{M}$ & 90 & Jaundice & US,CT,MRI,PTC & Gallbladder cancer & Nonoperative, PTC & M. type I \\
\hline 5 & $\mathrm{M}$ & 78 & Jaundice & US,CT & Klatskin's tumor & Cholecystectomy & M. type I \\
\hline 6 & $\mathrm{M}$ & 61 & Jaundice & US,CT,MRI,ERCP & Mirizzi & Cholecystect. + T-tube & Mirizzi type II \\
\hline 7 & $\mathrm{~F}$ & 65 & Jaundice & US,MRI,ERCP & Mirizzi & Cholecystectomy & M. type I \\
\hline 8 & $\mathrm{M}$ & 72 & Jaundice & US,ERCP & Gallbladder cancer & Hepatico-jejunostomy & Mirizzi type II \\
\hline 9 & $\mathrm{M}$ & 67 & Pain & US,CT,MRI,ERCP & Mirizzi & Cholecystectomy & M. type I \\
\hline 10 & $\mathrm{~F}$ & 65 & Jaundice & US,CT,ERCP & Mirizzi & Cholecystectomy & M. type I \\
\hline 11 & $\mathrm{~F}$ & 38 & Jaundice & US,CT,MRI,PTC & Gallbladder cancer & Hepatico-jejunostomy & Mirizzi type II \\
\hline 12 & $\mathrm{M}$ & 68 & Jaundice & US,CT,MRI,ERCP & Mirizzi & Explor.Laparotomy & M. type I + GBC \\
\hline 13 & $\mathrm{~F}$ & 56 & Jaundice & US,CT,ERCP & Mirizzi & Cholecystect. + T-tube & Mirizzi type II \\
\hline 14 & $\mathrm{~F}$ & 56 & Pain & US,CT,ERCP & Mirizzi & Cholecystect. + T-tube & Mirizzi type II \\
\hline 15 & $\mathrm{~F}$ & 56 & Jaundice & US,CT,MRI & Gallbladder cancer & Hepatico-jejunostomy & Mirizzi type II \\
\hline 16 & $\mathrm{M}$ & 68 & Jaundice & US,CT,ERCP & Gallbladder cancer & Cholecystectomy & M. type I \\
\hline 17 & M & 25 & Jaundice & US,MRI,ERCP & Mirizzi & Cholecystectomy & M. type I \\
\hline 18 & $\mathrm{~F}$ & 52 & Jaundice & US,MRI,ERCP & Mirizzi & Right hepatectomy & M. type I + GBC \\
\hline
\end{tabular}

Note. US=ultrasonography; $\mathrm{CT}=$ Computerized Tomography; $\mathrm{MRI}=$ magnetic resonance imaging; $\mathrm{PTC}=$ percutaneous transhepatic cholangiography; $\mathrm{ERCP}=$ endoscopic retrograde cholangiopancreatography; $\mathrm{GBC}=$ gallbladder cancer.

Percutaneous cholangiography was performed in 2 patients and a percutaneous drainage was placed preoperatively. After diagnostic investigations, a diagnosis of Mirizzi syndrome was achieved in 11 patients, a diagnosis of gallbladder cancer was achieved in 6 patients, and a Klatskin tumor was diagnosed in one patient.

Seventeen patients underwent surgery. Eight patients with type I Mirizzi syndrome underwent a simple cholecystectomy, leaving in place the portion of the infundibulum adherent to the CBD. In three cases of Mirizzi type II, after cholecystectomy, a bile duct repair over T-tube was made. In other three cases of Mirizzi type II, an excision of the gallbladder and involved CBD with hepaticojejunostomy on Roux-en-Y loop was performed. The patient with type II Mirizzi presenting with acute colangitis was treated urgently with biliary drainage by ERCP and two months later underwent cholecystectomy and hepatico-jejunostomy. In patients with preoperative erroneous diagnosis of gallbladder cancer or Katskin's tumor, frozen sections and definitive histological examination clarified the diagnosis of Mirizzi syndrome. On the contrary, an unexpected gallbladder cancer associated with the Mirizzi syndrome was diagnosed intraoperatively in two patients: a 68-year-old man who received an explorative laparotomy for an unresectable cancer with peritoneal carcinomatosis and a 52-year-old female who underwent right hepatectomy with $\mathrm{CBD}$ excision for gallbladder cancer involving the CBD. Finally, a 90-year old man, who had initially received a diagnosis of gallbladder cancer, was reevaluated with CT scan and MRI eighteen months later and was diagnosed as Mirizzi type-I syndrome. This patient did not undergo surgery and was treated by percutaneous drainage.
The overall morbidity rate was $16.6 \%$ (one case of postERCP pancreatitis and two cases of wound infection after surgery). There was no postoperative mortality. The 8 patients who underwent simple cholecystectomy enjoy good health with normal liver function tests. The three patients with CBD reconstruction over T-tube developed cholestasis after T-tube removal, due to the occurrence of a postoperative stricture: all cases were successfully treated with ERCP and multiple plastic stents insertions. The 4 patients who had undergone hepaticojejunostomy enjoy good health with normal liver function tests. The patient with unexpected finding of gallbladder cancer died three months after surgery. The patient who underwent right hepatectomy is alive six months after surgery and is being treated with chemotherapy. The patient with incorrect initial diagnosis of gallbladder cancer enjoys good health and replaces his percutaneous drainage at three month intervals. Clinical data are summarized in Table 1. All patients were monitored every six months after surgery (mean follow-up: 24 months, range: 6-48) by clinical evaluation, blood tests, and ultrasounds.

\section{Discussion}

The review of this clinical experience on Mirizzi syndrome allows some considerations regarding the diagnosis and treatment of the disease. In this series, patients with Mirizzi type I represented the majority of cases $(11 / 18=61 \%)$. Preoperative diagnosis was correct in 11 cases out of $18(61 \%)$, while the unexpected finding of cancer occurred in two cases (11\%) a significant percentage, considering that unexpected cancer is usually found in less than $1 \%$ of cholecystectomies [11]. However, there is a complex relationship between Mirizzi 


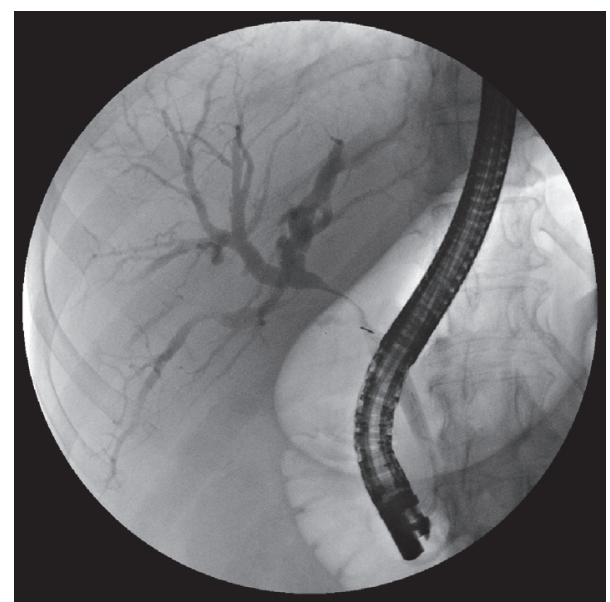

Figure 2: ERCP in a case of Mirizzi type I: note the smooth and regular stricture.

syndrome and cancer: the high incidence of unexpected cancer is confirmed by the literature, as well as the erroneous preoperative diagnosis of cancer consequent to the particular clinical presentation $[12,13]$. In our cases, a false diagnosis of cancer occurred in $39 \%$ of cases (7/18) explainable by the onset of jaundice in the absence of painful symptoms. The risk of bile duct injury during cholecystectomy for Mirizzi syndrome is increased according to the literature [14]. This fact is consequent to the anatomical difficulties during the dissection of the Calot's triangle.

Diagnostic difficulties are explained by the different clinical presentations of the disease. The classic presentation is obstructive jaundice without painful symptoms and with evidence, at ultrasonography, of a gallstone impacted in the gallbladder infundibulum and determining an external obstruction of the CBD with consequent dilation of the intrahepatic biliary tree. The diagnosis needs to be confirmed by CT or MRI and, finally, by surgery with histological examination. In these cases, in which it may be a Mirizzi syndrome type I or type IIa according to Béltran classification [10], it is suggested to perform an ERCP with the placement of one or more biliary plastic stents that simplify the surgical procedure by providing a protection to the CBD. Simple nasobiliary drainage does not eliminate this necessity. In addition, direct cholangiography obtained by ERCP provides a significant contribution to the correct diagnosis (Figures 2, 3,4 , and 5 ).

In type I Mirizzi syndrome, surgical treatment involves removal of the gallbladder leaving in place the portion of the infundibulum adherent to the CBD. In this way, the patency of the CBD is ensured by the presence of the stents. In type IIa Mirizzi syndrome the gallbladder is partially removed and part of the infundibular wall is used for the closure of the CBD. After surgery, stents can be eliminated spontaneously or subsequently removed. This modality of treatment is preferable to the CBD reconstruction over Ttube. In fact, after the removal of the T-tube, a stricture can appear requiring further endoscopic treatment, as in three cases of this series. The 4 cases of Mirizzi type IIb in

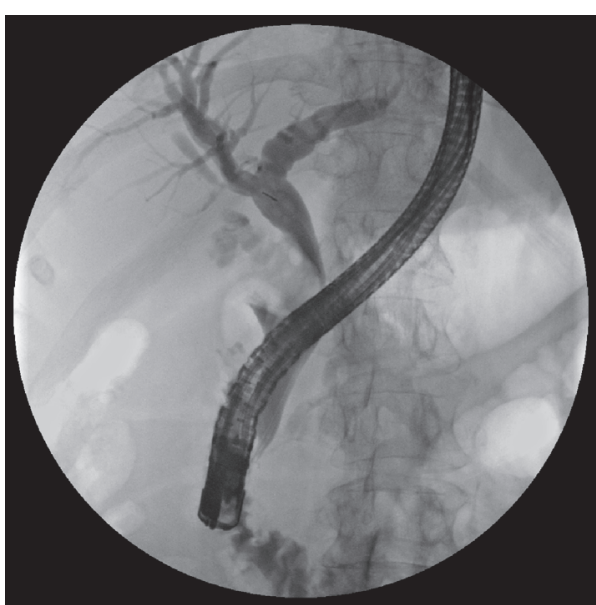

FIGURE 3: ERCP in a case of Mirizzi type II: filling defect of the CBD caused by a big stone.

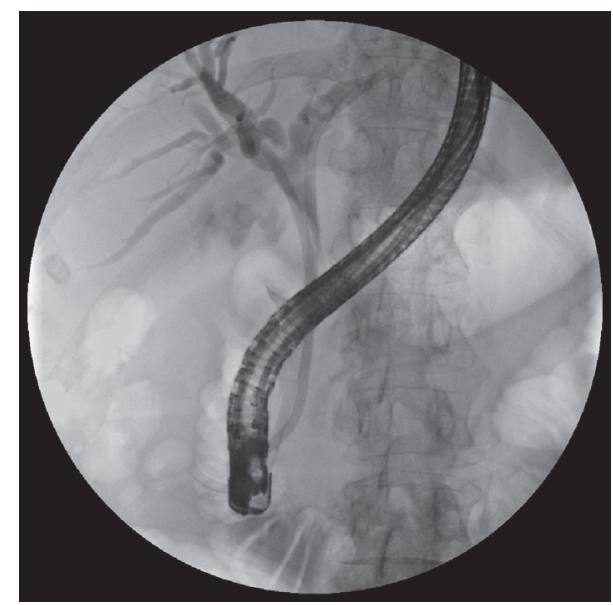

FIGURE 4: The same case after insertion of 2 stents.

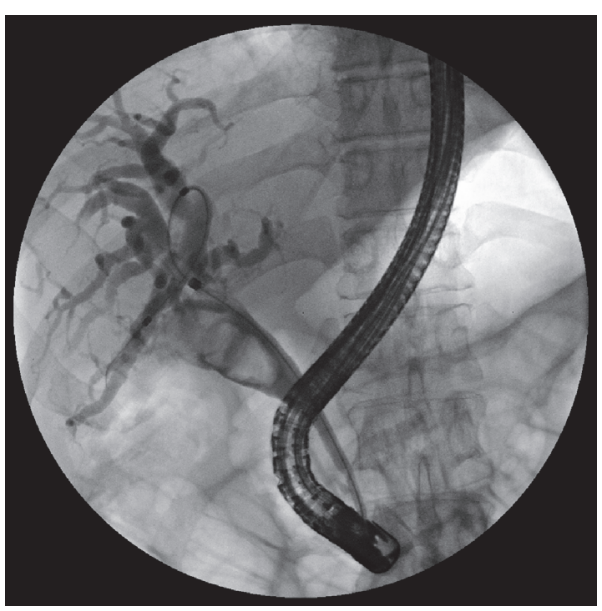

FIGURE 5: ERCP in a case of Mirizzi type II: there is a "common cavity" involving the gallbladder and the CBD. 


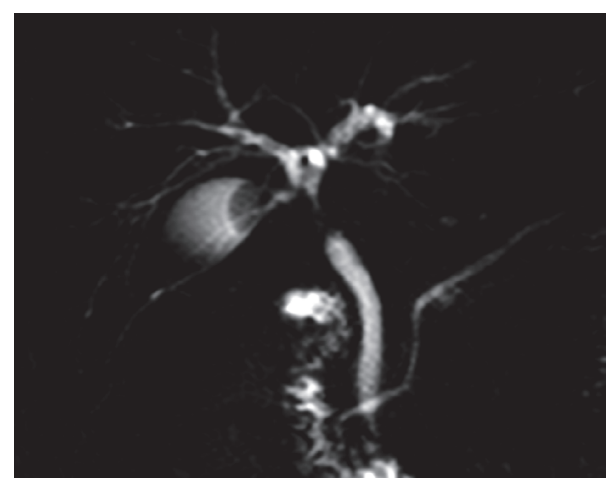

FIGURE 6: MRI cholangiography in a patient with Mirizzi type I mimicking a biliary cancer.

our series received hepaticojejunostomy with an uneventful clinical outcome at follow-up. Regarding laparoscopic surgical approach, there are different opinions in the literature. Although laparotomy is considered a safer approach in the management of patients with Mirizzi syndrome, some authors $[15,16]$ report satisfactory results with laparoscopic technique, performing a subtotal cholecystectomy. However, the laparoscopic approach is recommended only to very experienced surgeons.

Another frequent clinical picture of the Mirizzi syndrome is that of a stricture mimicking biliary cancer. When the associated inflammatory process is predominant, it involves the $\mathrm{CBD}$ and mimics a neoplastic stricture at the MRcholangiography (Figure 6). Patients usually undergo preoperative ERCP with placement of one or more stents. Endobiliary biopsies are obviously negative, taking into account the high frequency of false-negative findings. The diagnosis is clarified during surgical exploration by personal experience of the surgeon and analysis of the frozen-sections specimens. In these cases, the clinical onset and MRI findings can justify the preoperative diagnostic error. It should also be considered that up to $15 \%$ of suspicious biliary strictures are postoperatively found to be benign after surgery for Klatskin tumor [17]; the Mirizzi syndrome is one of the possible causes of this specific, sometimes unavoidable, diagnostic error.

A clinical onset with acute cholangitis is typical of type II Mirizzi syndrome: in these cases, diagnosis is facilitated by ERCP that allows to reduce the pressure in the CBD by positioning of nasobiliary drains or stents. However, surgery still represents the definitive solution: the choice between cholecystectomy with fistula closure and hepaticojejunostomy should be evaluated by the surgeon taking into account the extent of the CBD involvement, according to the Béltran categories [10].

In some cases, the Mirizzi syndrome can represent an unexpected intraoperative finding and the main risk is accidental bile duct injury. This happens when the surgeon wants to complete the cholecystectomy "at any cost" without previous identification of the CBD. On the contrary, the main goal must be to avoid any injury to the bile ducts and, therefore, the placement of a cholecystostomy may be an appropriate and justified measure as a bridge solution before referring the patient to a hepatobiliary center. Alternatively, it may be carried out a subtotal cholecystectomy, leaving in place a portion of the infundibulum adherent to the CBD, after removing the stones. Testini and coworkers suggested a decision algorithm for emergency in nonspecialized centers [18].

\section{Conclusions}

Mirizzi syndrome continues to be a disease of difficult diagnosis and treatment. The general surgeon without long experience in hepatobiliary surgery should refer the patient to a specialized hepatobiliary surgical center. It is difficult to standardize the treatment of the disease since clinical presentation and anatomical situations are very variable (types I or II, suspected carcinoma). It is important, however, for surgeons to know the disease and the possible intraoperative challenging situations. In patients with Mirizzi syndrome type I, the best management seems to be the preliminary placement of one or more stents by ERCP, followed by cholecystectomy leaving in place a small portion of the gallbladder adherent to the CBD. It is advisable to leave the stent in the postoperative period and remove it after nearly 2 months. In the occurrence of a postoperative stricture, endoscopic treatment with positioning of multiple plastic stents is recommended until resolution. In Mirizzi syndrome type II subtype a (according to Béltran classification), treatment may be similar to type I; however, a close attention to the diameter of the residual $\mathrm{CBD}$ is required during the reconstructive phase. In type IIb, the definitive solution appears to be hepaticojejunostomy. As for the type of surgical approach (laparoscopy or laparotomy), laparoscopy is reserved to a very experienced surgeon. However, in our opinion, laparotomy allows better evaluation of biliary anatomy, avoiding any risk of bile duct injury and with the advantage of a more accurate surgical procedure.

\section{Data Availability}

All clinical data are listed in the table and are available for consultation.

\section{Conflicts of Interest}

The authors declare that they have no conflicts of interest.

\section{References}

[1] L. N. Leopardi and G. J. Maddern, "Pablo Luis Mirizzi: The man behind the syndrome," ANZ Journal of Surgery, vol. 77, no. 12, pp. 1062-1064, 2007.

[2] A. M. Maroll and L. E. Granero, "Pablo Luis Mirizzi," Acta Gastroenterológica Latinoamericana, vol. 39, pp. 177-178, 2009.

[3] P. L. Mirizzi, "Syndrome del conducto hepatico," Journal International de Chirurgie, vol. 8, pp. 731-733, 1948.

[4] C. K. McSherry, H. Ferstenberg, and M. Virshup, "The Mirizzi syndrome: suggested classification and surgical therapy," Surgical Gastroenterology, vol. 1, pp. 219-225, 1982. 
[5] A. Csendes, J. C. Diaz, P. Burdiles, F. Maluenda, and O. Nava, "Mirizzi syndrome and cholecystobiliary fistula: a unifying classification," British Journal of Surgery, vol. 76, no. 11, pp. 11391143, 1989.

[6] M. A. Beltran and A. Csendes, "Mirizzi syndrome and gallstone ileus: an unusual presentation of gallstone disease," Journal of Gastrointestinal Surgery, vol. 9, no. 5, pp. 686-689, 2005.

[7] C. A. Solis-Caxaj, "Mirizzi syndrome: Diagnosis, treatment and a plea for a simplified classification," World Journal of Surgery, vol. 33, no. 8, pp. 1783-1784, 2009.

[8] M. A. Beltran, "Reply," World Journal of Surgery, vol. 33, no. 8, pp. 1786-1787, 2009.

[9] M. A. Beltran, A. Csendes, and K. S. Cruces, "The relationship of Mirizzi syndrome and cholecystoenteric fistula: validation of a modified classification," World Journal of Surgery, vol. 32, no. 10, pp. 2237-2243, 2008.

[10] M. A. Beltrán, "Mirizzi syndrome: history, current knowledge and proposal of a simplified classification," World Journal of Gastroenterology, vol. 18, no. 34, pp. 4639-4650, 2012.

[11] G. Clemente, G. Nuzzo, A. M. de Rose et al., "Unexpected Gallbladder Cancer after Laparoscopic Cholecystectomy for Acute Cholecystitis: A Worrisome Picture," Journal of Gastrointestinal Surgery, vol. 16, no. 8, pp. 1462-1468, 2012.

[12] C. A. Redaelli, M. W. Büchler, M. K. Schilling et al., "High coincidence of Mirizzi syndrome and gallbladder carcinoma," Surgery, vol. 121, no. 1, pp. 58-63, 1997.

[13] T. L. V. D. Prasad, A. Kumar, S. S. Sikora, R. Saxena, and V. K. Kapoor, "Mirizzi syndrome and gallbladder cancer," Journal of Hepato-Biliary-Pancreatic Sciences, vol. 13, no. 4, pp. 323-326, 2006.

[14] K. Psarras, K. D. Ballas, T. E. Pavlidis et al., "A case of Mirizzi's syndrome mimicking carcinoma: the role of CBD-stenting for easy surgical management," Journal of Laparoendoscopic \& Advanced Surgical Techniques A, vol. 19, no. 4, pp. 513-516, 2009.

[15] D. Koike, Y. Suka, M. Nagai, Y. Nomura, and N. Tanaka, "Laparoscopic management of Mirizzi syndrome without dissection of Calot's triangle," Journal of Laparoendoscopic \& Advanced Surgical Techniques A, vol. 27, no. 2, pp. 141-145, 2017.

[16] H. Yuan, T. Yuan, X. Sun, and M. Zheng, "A Minimally Invasive Strategy for Mirizzi Syndrome Type II: Combined Endoscopic with Laparoscopic Approach," Surgical Laparoscopy Endoscopy \& Percutaneous Techniques, vol. 26, no. 3, pp. 248-252, 2016.

[17] B. Blechacz, "Cholangiocarcinoma: Current knowledge and new developments," Gut and Liver, vol. 11, no. 1, pp. 13-26, 2017.

[18] M. Testini, L. I. Sgaramella, G. M. De Luca et al., "Management of Mirizzi Syndrome in Emergency," Journal of Laparoendoscopic \& Advanced Surgical Techniques, vol. 27, no. 1, pp. 28-32, 2017. 


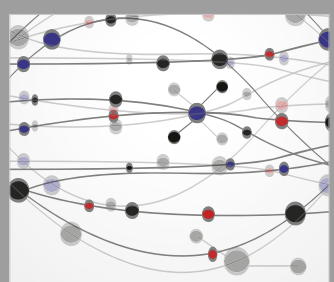

The Scientific World Journal
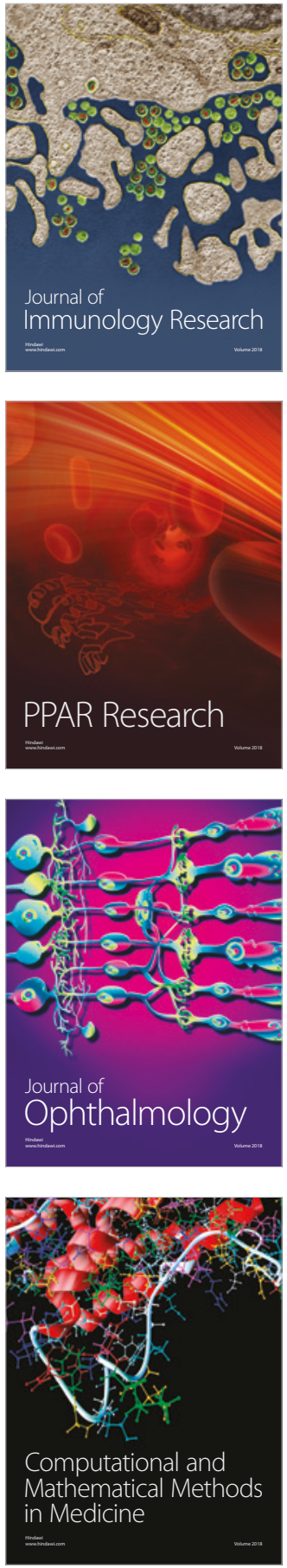

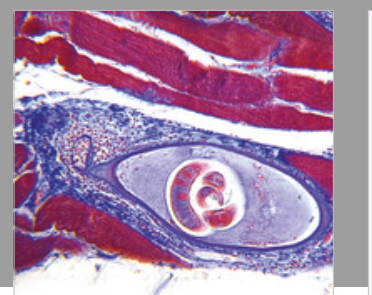

Gastroenterology Research and Practice

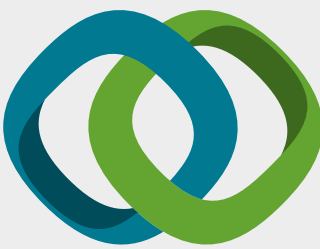

\section{Hindawi}

Submit your manuscripts at

www.hindawi.com
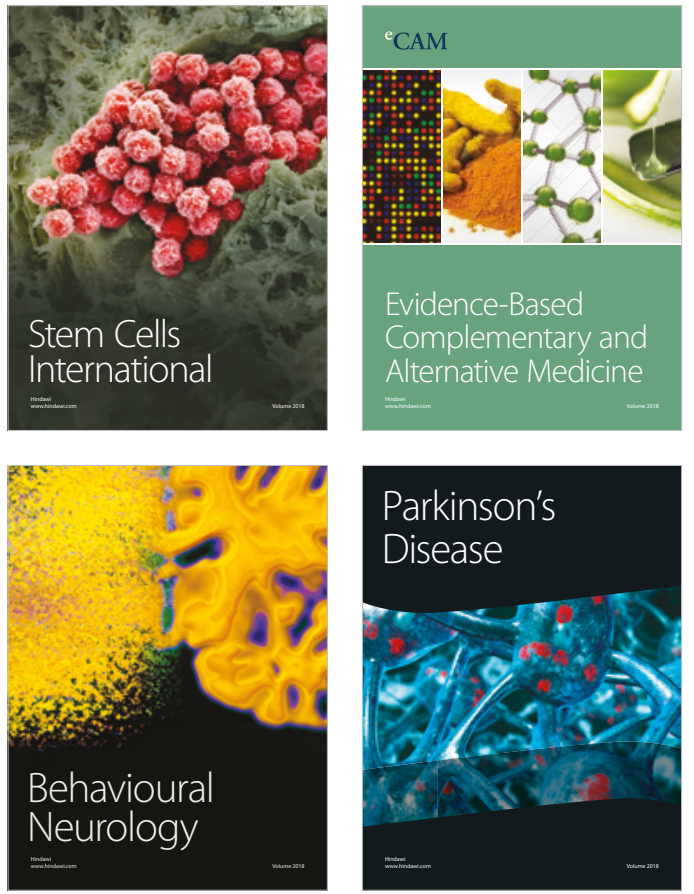

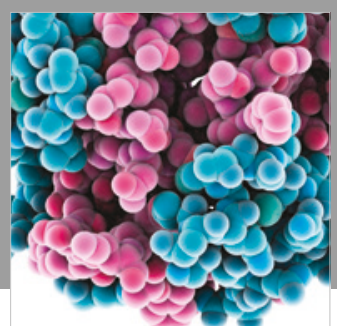

ournal of

Diabetes Research

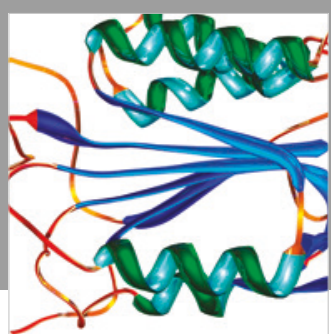

Disease Markers
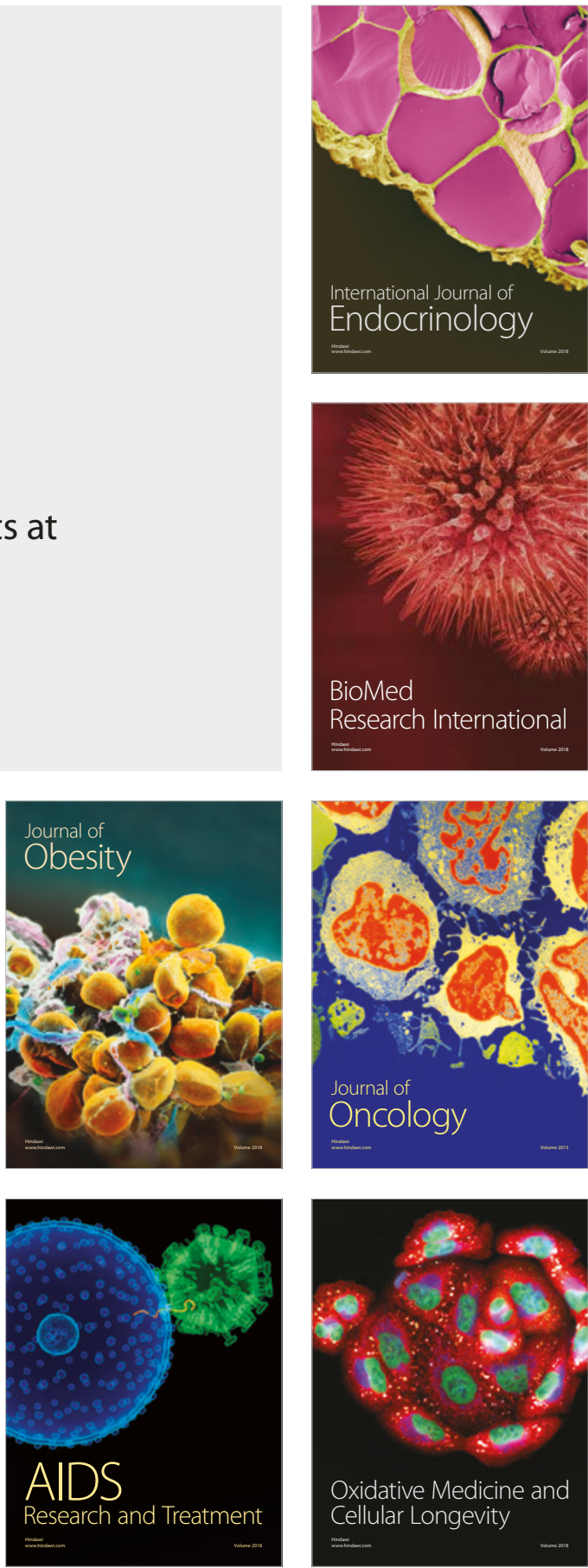